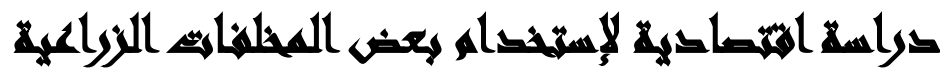

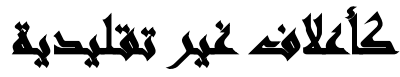

[11]

$$
\begin{aligned}
& \text { فاروق محمد التلاوي(')- نيرة يحيى سليمان(')- سهام أحمد عبد الحميد(') } \\
& \text { أحمد عبد القادر خليف }
\end{aligned}
$$

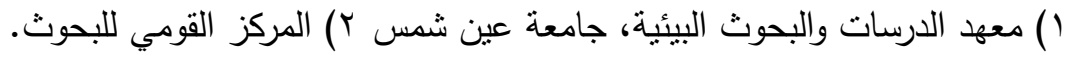

\section{المستخليك}

تتاولت الدراسة تطور أعداد الماثية على مستوى جمهورية مصر العربية ومحافظة

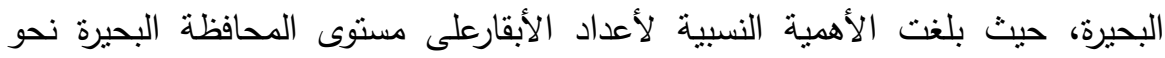

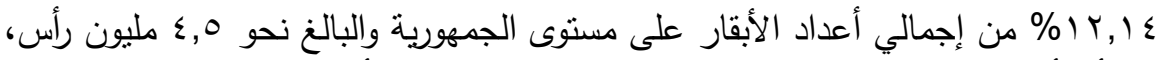

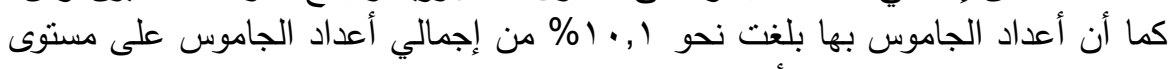

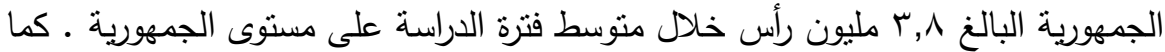

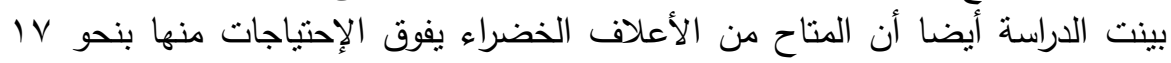

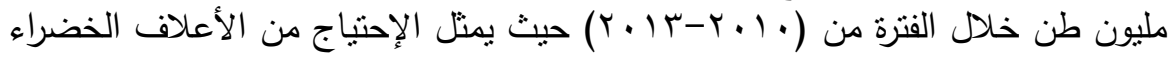

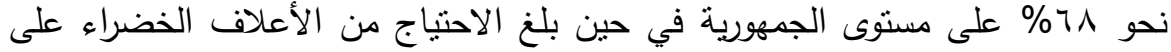

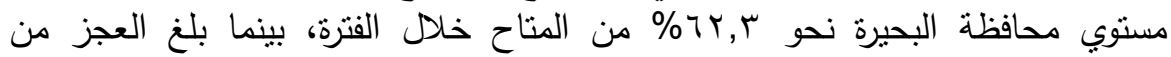

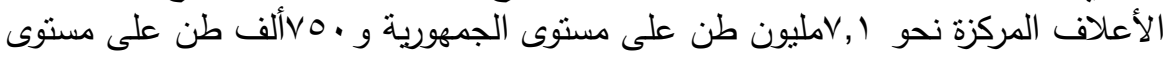

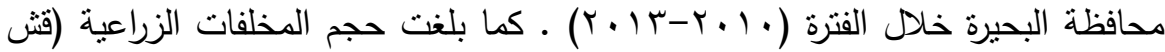

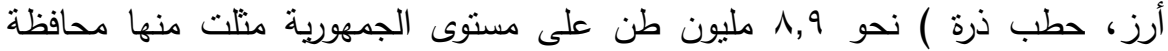

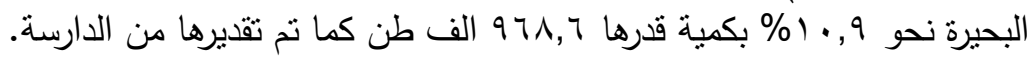

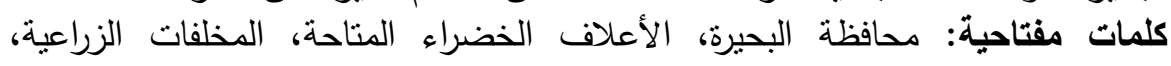
احتياجات حيوانات المزرعة. 
مجلة العلوم البيئية

معهد الدراسات والبحوث البيئية - جامعة عين شمس البه

\section{ravall}

يعتبر الطلب على الاعلاف مشتق من الطلب على المنتجات الحيوانية \} لحوم -

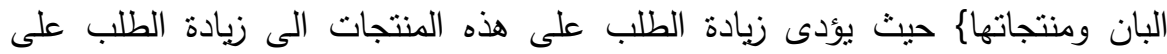
الاعلاف لذا تتوقف تتمية الانتاج الحيوانى الى حد كبير على مدى توافر الاعلاف

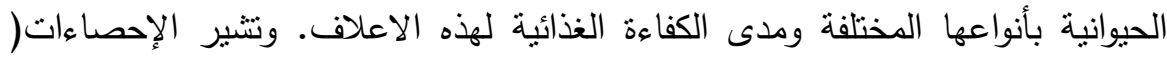

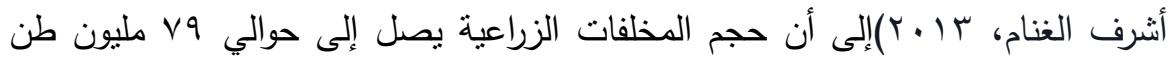

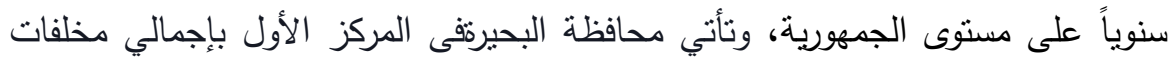

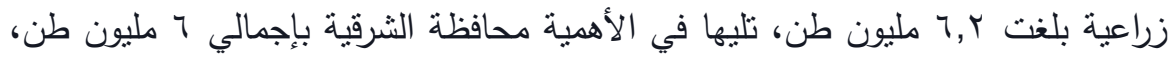
لذلك تحتل محافظة البحيرة أهمية نسبية بين محافظات الجمهورية في الإنتاج النباتي

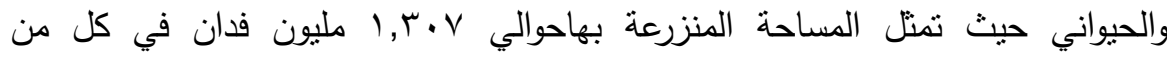

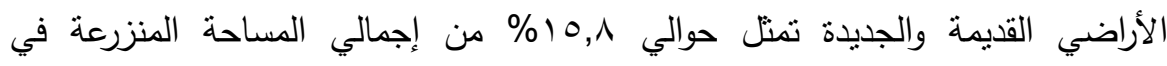

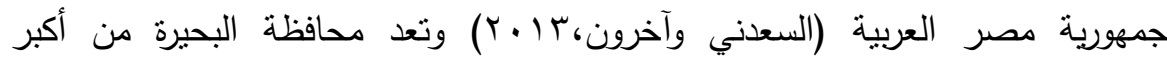
المحافظات ذات الأهمية النسبية في إنتاج المحاصيل التي تعتبر مصدراً للمخلفات

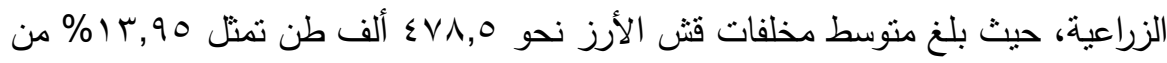

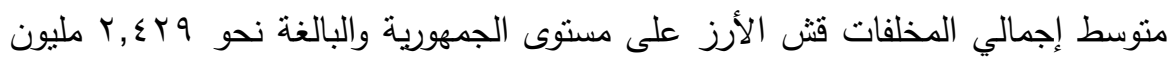

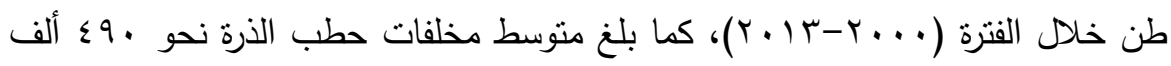

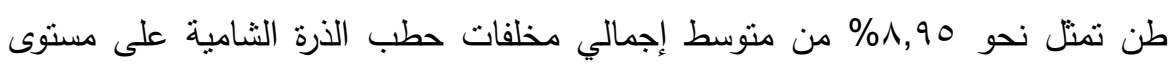

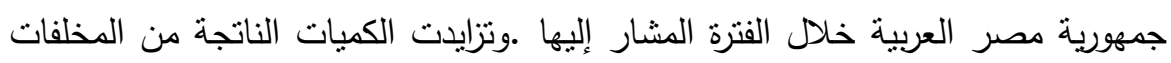
الزراعية بمختلف أنواعها زيادة كبيرة ارتبطت بالتوسع الكبير في العقود الأخيرة في زراعة

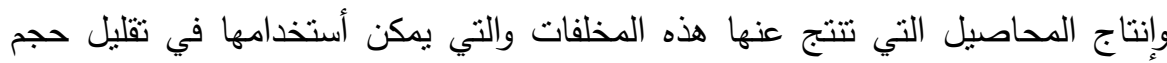
الفجوة العلفية بأستخدامها كأعلاف غير تقليدية، ومن ثم المساهمة في توفير نصيب الفرد من البروتين الحيواني الذي يؤدي بدوره في احتفاظ الإنسان بصحته وحيويته وقدرته على بلى

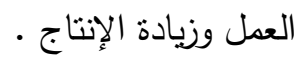




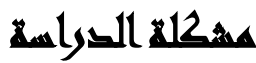

بالرغم من توفر كميات كبيرة من المخلفات الزراعية تعانى مصر من نقص مواد العلف الحيواني وبالتالى تتجه مصر لاستنيراد نسبة كبيرة منها سنوياً لسد الفجوة العلفية وتعتبر الأعلاف الحيوانية محدداً رئيسياً للإنتاج الحيواني ويلاحظ أن المتاح من الأعلاف لئه

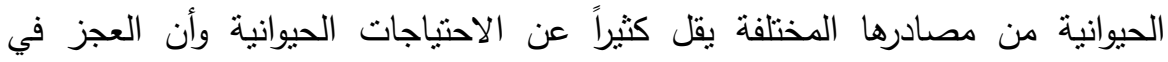
الموازنة العلفية يميل إلى التزايد سنة بعد أخرى.

\section{همبه هالدواسمة}

استهدفت الدارسة التعرف على الوضع الراهن للإحتياجات العلفية للحيوانات المزرعية على مستوى الزراعة المصرية ومحافظة البحيرة في ضوء تطور أعدادها وتقدير

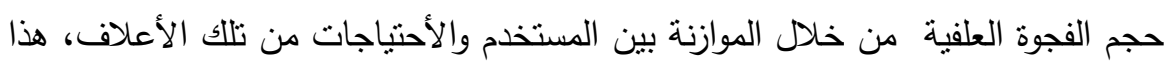

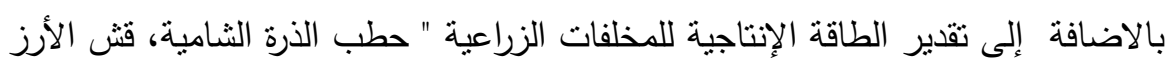
" في الزراعة المصرية بصفة عامة ومحافظة البحيرة بصفة خاصة.

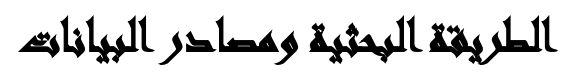

استخدمت الدراسة الطريقة الوصفية والكمية وذللك باستخدام معادلات الإتجاة الزمني العامواعتمدت الدراسة على البيانات الثانوية المنشورة وغير المنشورة كما اعتمد البحث البه

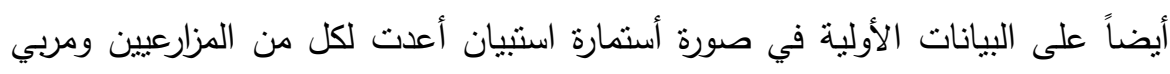
الأبقار والجاموس بمحافظة البحيرة.

\section{عبنة الدراسة}

تحتل محافظة البحيرة أهمية نسبية بين محافظات الجمهورية في الإنتاج النباتي والحيواني حيث تعد محافظة البحيرة من أكبر المحافظات ذات الأهمية النسبية في إنتاج المحاصيل التي تعتبر مصدرا للمخلفات الزراعية، حيث بلغ متوسط مخلفات فش الأرز نحو

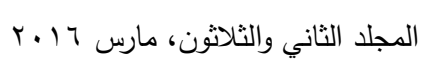




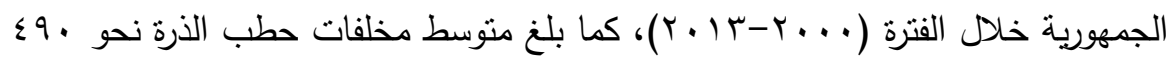
ألف طن تمنل نحو 9\% من منوسط إجمالي مخلفات الحطب على مستوى الجمهورية . وتم أختيار عنية عشوائية من القرى التابعة لمركز أبو حمص لانه يمثل أكبر نسبة من من

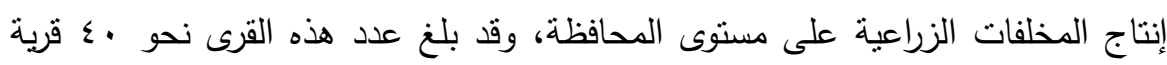
وذلك استتاداً لبيانات الإجارة الزراعية بمركز أبو حمص، ونم أختيار قريتي بسنتاواي وبركة غطاس بمركز أبو حمص لتمثيل عينة الدراسة استتاداً إلى الأهمية النسبية لكل منها

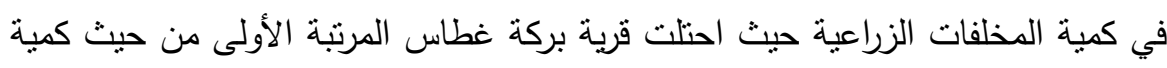

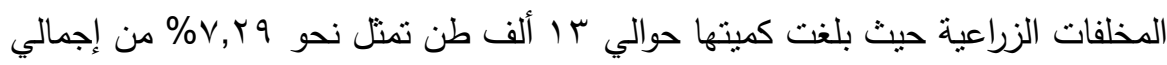
كمية المخلفات الزراعية بالمركز والبالغة حوالي ائ ألف طن، يليها قرية بسنتاواي بكمية

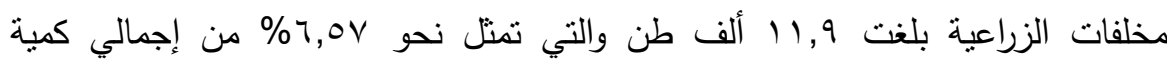

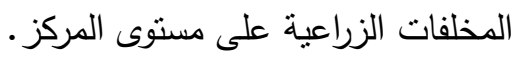

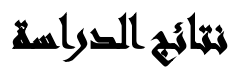

تتقسم الدراسة إلى ستة أجزاء، يهنم الجزء الأول بتطور أعداد الماثية بجمهورية

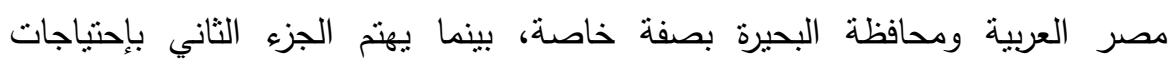
الحيوانات المزرعية من الأعلاف ومقدار المناح منها على مستوى الجمهورية ومحافظة البحيرة، في حين يهنم الجزء الثالث بالمخلفات الزراعية ودورها في تقليص حجم الفجوة

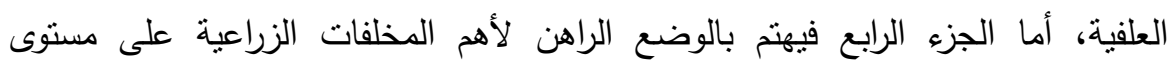

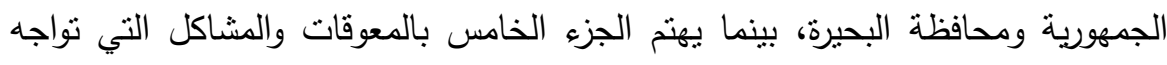

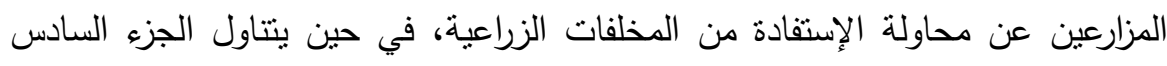

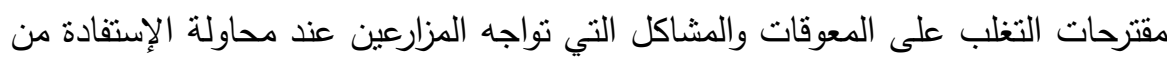
المخلفات الزراعية. أولاً : تطور أعداد الماثبية بجمهورية مصر العربية ومحافظة البحيرة: 
تتمثل الثروة الحيوانية في مصر في كل من الأبقار والجاموس والأغنام والماعز

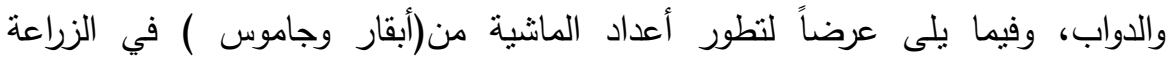

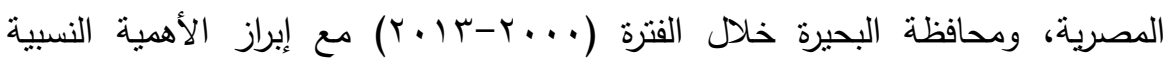
ل المحافظة البحيرة في كل من: أ- الأبقار: تحتل محافظة البحيرة المرتبة الأولى بين محافظات الجمهورية في أعداد

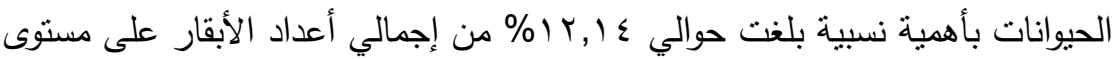

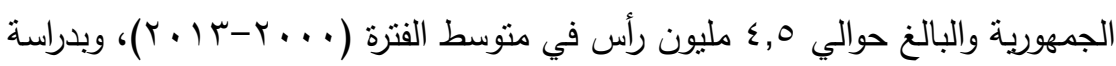
تطور أعداد الأبقار يتضح من الجدول رقم (1) أنها تزايدت على مستوى الجمهورية رئية

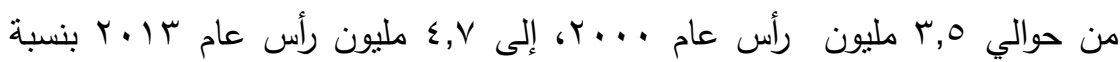

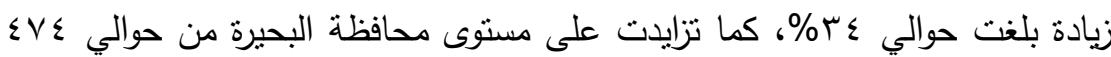

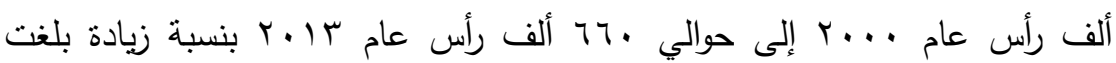
حوالي 9\%\% . كما يتضح من نتائج معادلات الاتجاه العام الزمني لأعداد الأبقار

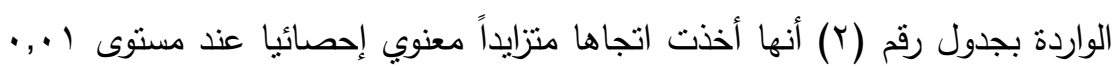

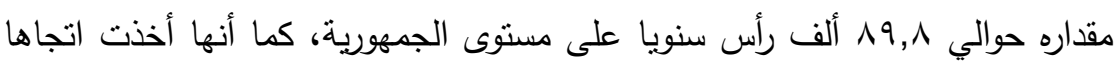
متزايدا غير معنوي احصائيا بلغ حوالي 9 1, ألف رأس على مستى مستوى محافظة البحيرة،

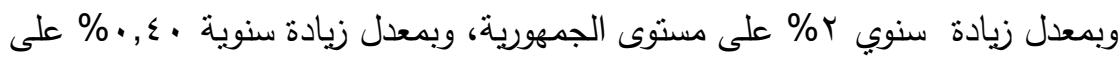
مستوى محافظة البحيرة . ب- الجاموس: تحتل محافظة البحيرة المرتبة الأولى بين محافظات الجمهورية في أعداد

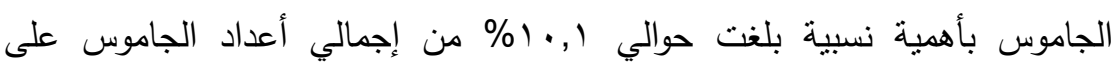

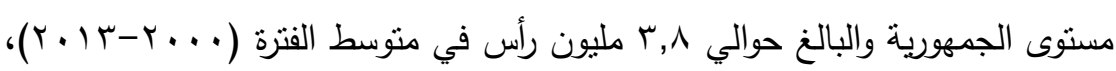

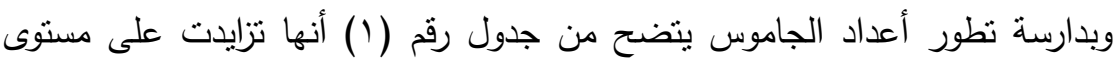

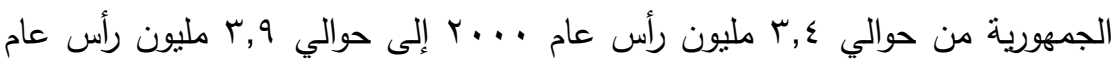

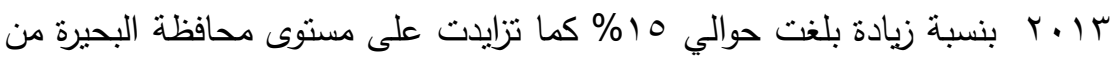

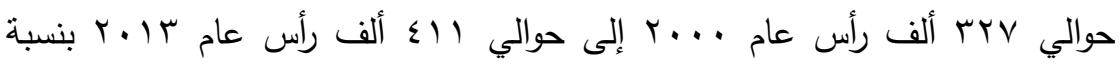

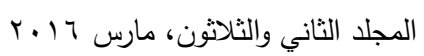


زيادة بلغت حوالي 0\% \% م كما يتضح من نتائج معادلات الاتجاه العام لأعداد

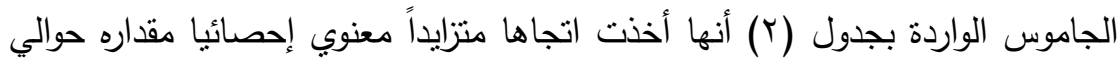

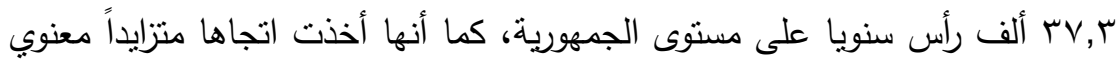
واحصائيا بلغ حوالي \, \ ألف رأس على مستوى محافظة البحيرة، وبمعدل زيادة

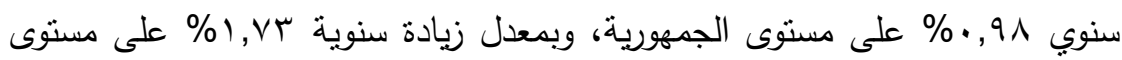
محافظة البحيرة، ويتضح من ذلك زيادة معدل النمو السنوي لأعداد الجاموس بالبحيرة

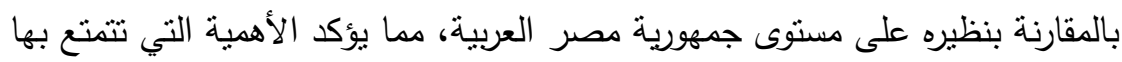

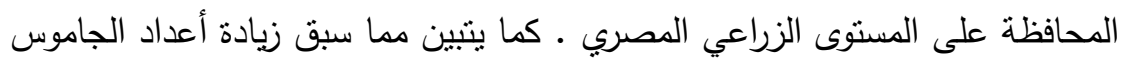

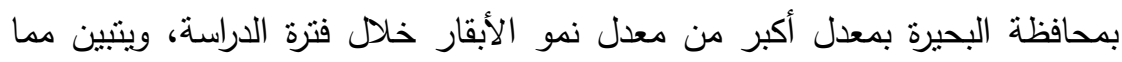

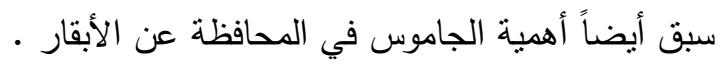
جدول رقـم(1): تطور اعدادالأبقار والجـاموس في مصر بمحافظـة البحيرة خـلال الفترة

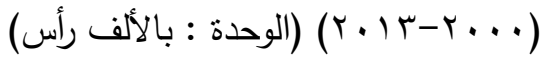

\begin{tabular}{|c|c|c|c|c|c|c|}
\hline \multirow{2}{*}{$\%$} & \multicolumn{2}{|c|}{ الجاموس } & \multirow{2}{*}{$\%$} & \multicolumn{2}{|c|}{ الأبقار } & \multirow{2}{*}{ السنوات } \\
\hline & البحيرة & الجمهورية & & البحيرة & الجمهورية & \\
\hline 9,71 & TrV & rrvq & $1 T, \varepsilon \Gamma$ & $\varepsilon \vee \leqslant$ & ror. & $r \ldots$ \\
\hline 9,71 & $T \leqslant Y$ & rorr & $1 \Gamma, \Gamma q$ & 0.9 & $r \wedge \cdot 1$ & r.. \\
\hline 9,7 & TOV & TVIV & $1 \pi, \Gamma$ & $0 \leqslant \Gamma$ & $\varepsilon \cdot \lambda r$ & $r \ldots r$ \\
\hline$q, \vee \leqslant$ & r4A & TVVV & $1 Y, \wedge 0$ & $0 \leqslant r$ & EYTV & $r \ldots r$ \\
\hline$q, \vee r$ & rV & $r \wedge \leqslant 0$ & $1 r, \wedge q$ & $07 r$ & $\varepsilon r 7 q$ & Y.. \\
\hline 9,99 & rAN & rA10 & $|r, 0|$ & 071 & $\varepsilon \leqslant 10$ & Y... \\
\hline 9,91 & rar & rarv & $\mid r, \varepsilon V$ & ovo & $\left.\sum 7\right)$. & $Y \ldots T$ \\
\hline $1 \cdot, r$ & $\sum 17$ & $\varepsilon \cdot \varepsilon r$ & IT,YY & $7 . r$ & हqस & $Y \ldots V$ \\
\hline $1 \cdot, r$ & 纟) & $\varepsilon .0 \%$ & 11,00 & $0 \wedge$ & $0 . Y \mu$ & $r \cdots \Lambda$ \\
\hline $1 \cdot, V$ & 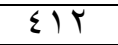 & rArq & $T K, \mu r$ & 001 & EOYo & $r \ldots 9$ \\
\hline $1 \cdot, r$ & rq1 & 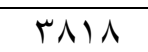 & $I Y, Y T$ & $0 \wedge$. & $\varepsilon V Y q$ & $r \cdot 1$. \\
\hline $1 \cdot, V$ & $\left.\sum Y\right\rceil$ & rqAr & $q, r q$ & $\leqslant \leqslant 9$ & $\sum \vee V q$ & $r \cdot 11$ \\
\hline $1 \cdot, r$ & ¿ro & ¿17 & $\Lambda, \uparrow$ & $\varepsilon Y \wedge$ & ¿9ะ7 & $r . M r$ \\
\hline $1 \cdot, 0$ & $\sum \prod$ & r910 & 15,9 & 77. & $\varepsilon V \leqslant \varepsilon$ & $T \cdot I T$ \\
\hline $1 \cdot, 1$ & 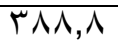 & $r \wedge \leqslant 9,1$ & 14,10 & $0 \leqslant \leqslant, V$ & $\varepsilon \leqslant \lambda \leqslant 0$ & المتوسط \\
\hline
\end{tabular}

المصدر: وزارة الزراعة واستصلاح الأراضي، قطاع الثئون الاقتصادية، الإدراة المركزية للاقتصاد الزراعي، نثرة إحصاءات الثروة الحيوانية، أعداد منقرقة. 
جدول رقم (ץ): الاتجاه العام لنطور أعداد الأبقار والجاموس على مستوى جمهورية مصر

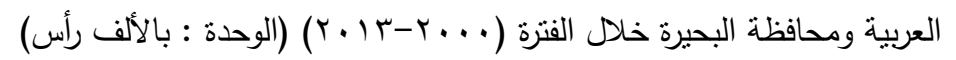

\begin{tabular}{|c|c|c|c|c|c|c|c|c|}
\hline الصالية & 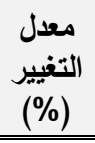 & المتوسط & ف & 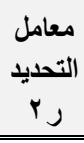 & 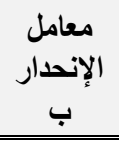 & أمعابلة & البيان & المعادلة \\
\hline خطية & r & $\varepsilon \leqslant 10$ & $r \cdot, \tau$ & $\cdot, V Y$ & $\begin{array}{l}\wedge 9, \wedge \\
(0,0)\end{array}$ & rᄉl., & بالجمهورية الأبقار & 1 \\
\hline خطية & $\cdot, \Sigma$. & $0 \leqslant \leqslant, V$ & $\cdot, Y V$ & $\cdot, \cdot r$ & $\begin{array}{c}r, 19 \\
(., 01)\end{array}$ & OYA & بالأبقارة & r \\
\hline خطية & $\cdot, 91$ & $r \wedge \leq q$ & 17,9 & $\cdot, 0 \wedge$ & $\begin{array}{l}r v, v \\
(\varepsilon, 1)\end{array}$ & rot7 & بالجمهورية & r \\
\hline خطية & 1,V & r人q & Or, & $\cdot, \lambda)$ & $\begin{array}{c}7, V \\
(V, r)\end{array}$ & r"ᄉ, r & الجالبحيرة & $\varepsilon$ \\
\hline
\end{tabular}

المصدر : جمعت وحسبت من بيانات جدول رقم ( ) ثانياً : احتياجات الحيوانات المزرعية من الأعلاف ومقدار المتاح منها على مستوى ردي الجمهورية ومحافظة البحيرة :

لتقدير احتباجات الحيوانات المزرعية أمكن تحويل أعداد الحيوانات المزرعية المختلفة إلى وحدات حيوانية مكافئة حنى يسهل تقدير الإحتياجات السنوية من الأعلاف الحيوانية،

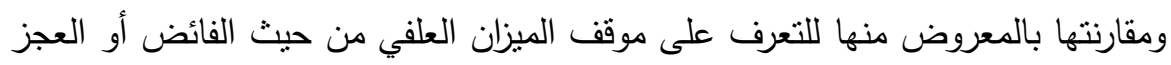

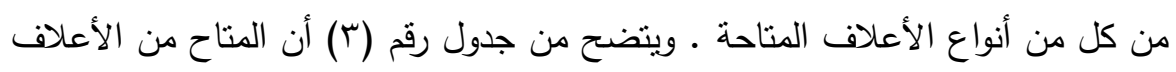

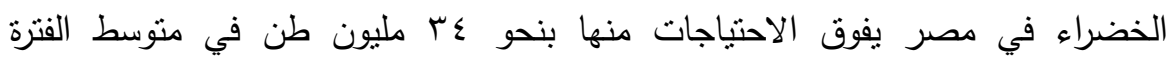

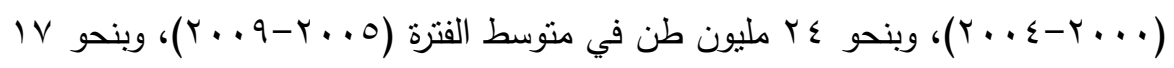

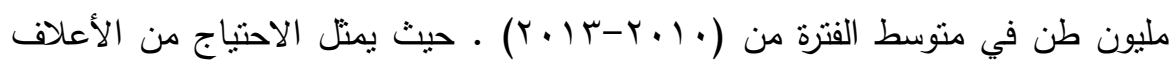

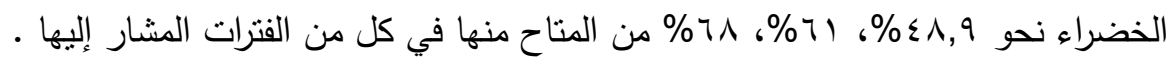

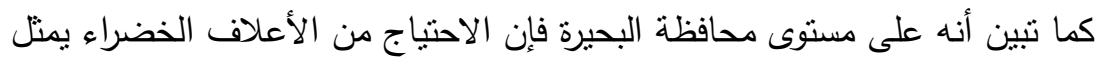

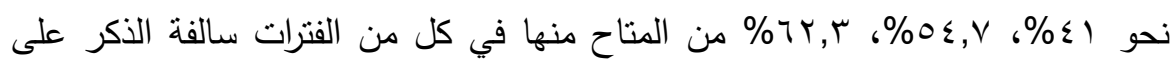

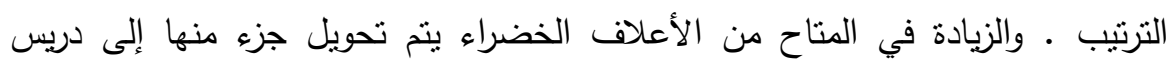

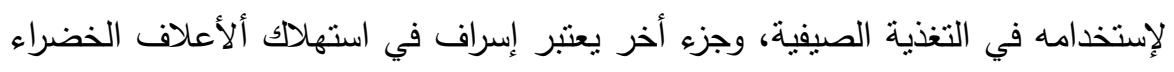

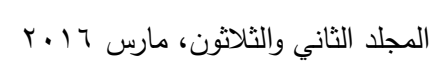


على مستوى الجمهورية و كذلك محافظة البحيرة . كما يتضح من جدول (r) أن

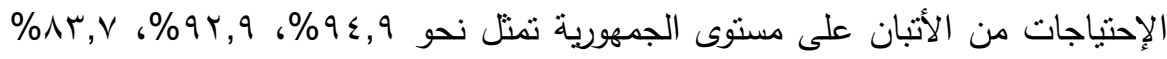

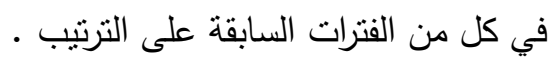

وعلى مستوى محافظة البحيرة فإن الإحتياجات من الإتبان فاقت المتاح منها خلاد

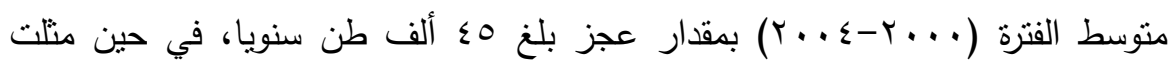

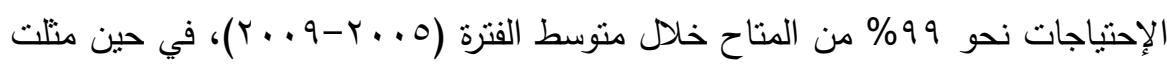

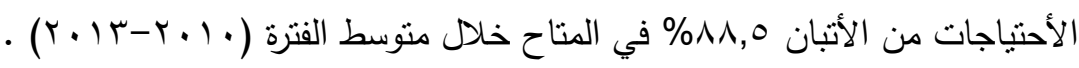
وبمقارنة الإحتياجات من المتاح من الأعلاف المركزة على مستوى الجمهورية خلان

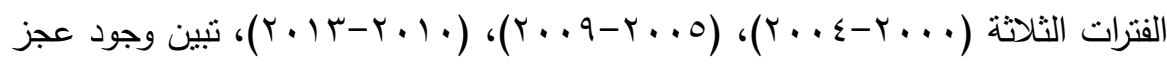

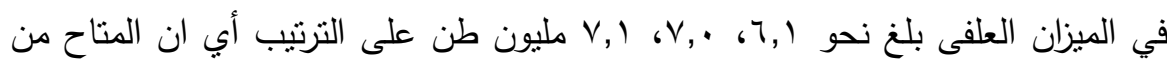

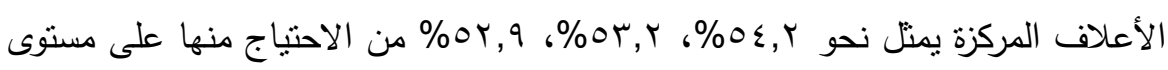
الجمهورية في كل منها على الترتيب ـ كما أن المتاح على مستوى محافظة البحيرة يمنل • . 
جدول رقـم (ץ): احتياجـات الحيوانـات المزرعيـة مـن الأعـلاف الحيوانيـة والمتـاح منهـا

والميزان العلفي على مستوى جمهوريـة مصر العربية، وكذللك محافظة

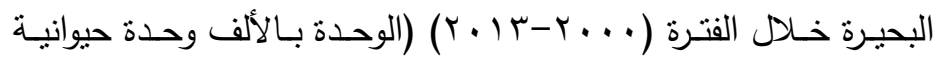

للحيوانات، وبالألف طن للأعلاف)

\begin{tabular}{|c|c|c|c|c|}
\hline \multicolumn{3}{|c|}{ متوسط الفترات الثلاث } & \multirow{2}{*}{ البيان } & \multirow[b]{2}{*}{ r } \\
\hline$(r+1 r-r+. q)$ & $(r+q-r+\cdots)$ & $(r \ldots \varepsilon-r \ldots)$ & & \\
\hline & & & جمهورية مص & \\
\hline$r \ldots 11,0$ & $19 \vee 97, \wedge$ & $|\vee 7| \cdot, \varepsilon$ & عدد الوحدات الحيوانية & 1 \\
\hline rᄉ..r,r & rVTAT & rosv, & الأحتياجات من الأعلاف & r \\
\hline $91 \times 9, V$ & $9.0 Y, 7$ & $\Lambda .09, \varepsilon$ & الإحتباجات من الآتبان & $r$ \\
\hline $101 \mathrm{VA,Y}$ & $10 \cdot 0 \cdot, r$ & 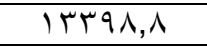 & الأحتياجات من الأعلاف المركزة & $\varepsilon$ \\
\hline $00 \leqslant \leqslant \mu, 0$ & $717 \cdot 9, Y$ & $7 \wedge 0 \leqslant \leqslant, 9$ & المتاح من الإعلاف الخضراء & 0 \\
\hline $1 \cdot 9 \cdot 0,1$ & $q \vee r \varepsilon, \varepsilon$ & $\Lambda \Sigma \wedge \vee$ & المتاح من الآتبان & 7 \\
\hline$\Lambda \cdot r \wedge, q$ & $\wedge \cdot 17, \lambda$ & VYฯA, r & المتاح من الأعلاف المركزة & $\mathrm{V}$ \\
\hline$I V \varepsilon \varepsilon \cdot, r$ & $r r q r V, r$ & $r \leqslant 99 \vee, 7$ & الميزان العلفي من الأعلاف & $\wedge$ \\
\hline $1 V \vee 7$ & $7 \wedge 1, \wedge$ & $\varepsilon Y V, T$ & الميزان العلفي من الأتبان & 9 \\
\hline$(\vee \backslash \leq ৭, Y-)$ & $(V \cdot r r, Y-)$ & $(7) \Gamma \cdot, 7-)$ & الميزان العلفي من الأعلاف & ! \\
\hline & & & محافظة البحيرة & \\
\hline 1101,0 & $119 \varepsilon, 1$ & $1 \cdot \wedge \wedge, \wedge$ & عدد الوحدات الحيوانية & 1 \\
\hline 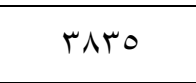 & 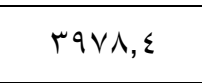 & rצro,^ & الأحتياجات من الآعلاف & $r$ \\
\hline 941 & 900,1 & $\Lambda \vee \backslash, Y$ & الإحتياجات من الآتبان & $r$ \\
\hline 1041,0 & $10 \wedge 9, Y$ & $1 \leqslant \leqslant \Lambda, Y$ & الإحتياجات من الأعلاف المركزة & $\varepsilon$ \\
\hline 7101 & VYVY, & $\wedge \wedge \leqslant 0$ & المتاح من الآعلاف الخضراء & 0 \\
\hline $1 \cdot \varepsilon \cdot, 0$ & $909, Y$ & $\wedge Y \neg, r$ & المتاح من الآتبان & 7 \\
\hline$\nabla \wedge I, Y$ & 097,7 & $\varepsilon r \tau, 7$ & المتاح من الأعلاف المركزة & $\mathrm{V}$ \\
\hline TIוT & ruq & or $19, r$ & الميزان العلفي من الأعلاف & $\wedge$ \\
\hline $119, r$ & $\mu, \mu \wedge$ & $(\varepsilon \varepsilon, \wedge-)$ & الميزان العلفي من الآتبان & 9 \\
\hline$\left(\vee 0 \cdot, 0^{-}\right)$ & $(99 \nvdash, \vee-)$ & $(1 \cdot 11,7-)$ & الميزان العلفي من الأعلاف & ! \\
\hline
\end{tabular}

المصدر: وزارة الزراعة واستصلاح الأراضي قطاع الثئون الإقتصادية الإحصاءات الزراعية

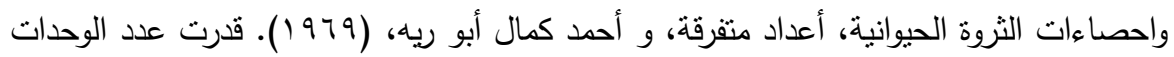

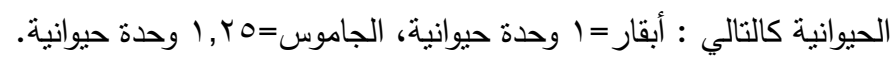

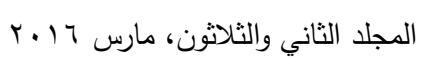


ثُالثاً : المخلفات الزراعية ودورها في تقليص حجم الفجوة العلقية: في ضوء عجز المناح من الأعلاف عن تلبية الإحتياجات الغذائية للحيوانات

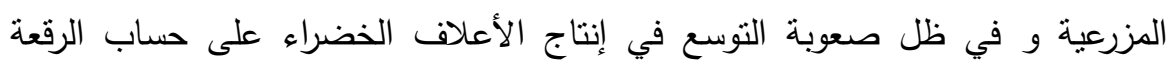

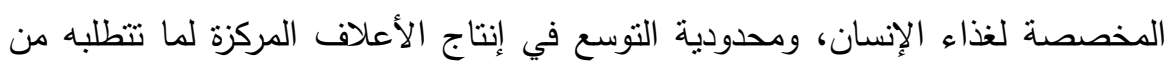

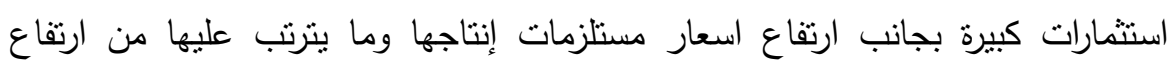
تكاليف التغذية عليها كان لابد من البحث عن مصادر جديدة لسد هذا العجز من

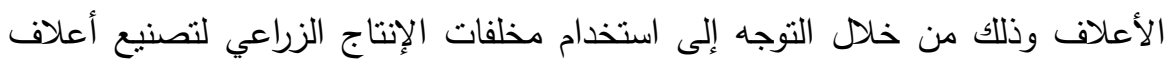
غير تقليدية تضاف كأعلاف مالئة لسد هذا العجز في الأعلاف.

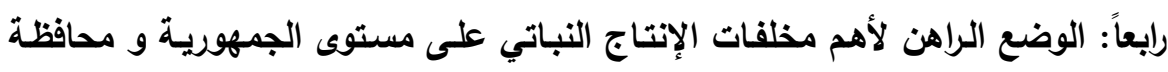
البحيرة: - (ابعا: تتمثل أهم مخلفات الإنتاج النباتي التي يمكن استخدامها كأعلاف غير تقليدية في

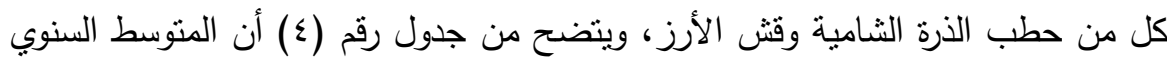

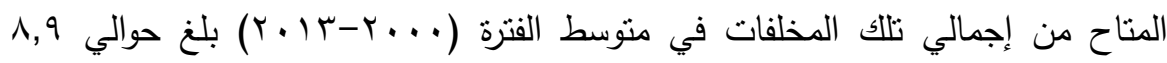

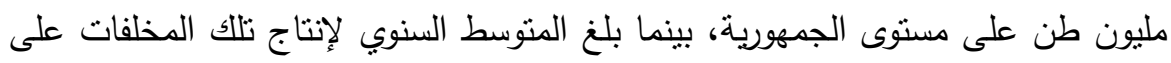

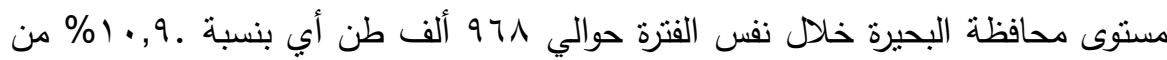

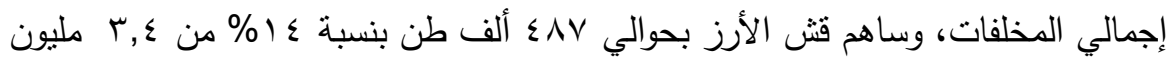

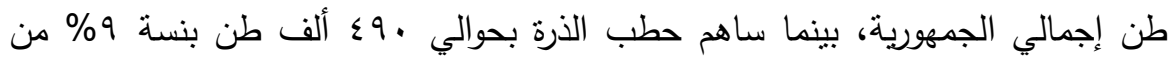
ـ,0 مليون طن إجمالي الجمهورية خلال منوسط الفترة سالفة الذكر ، حيث تجدر الإشارة

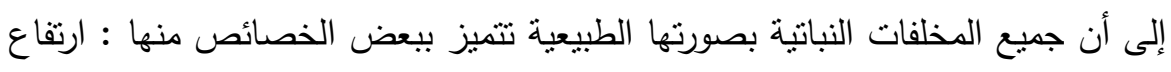

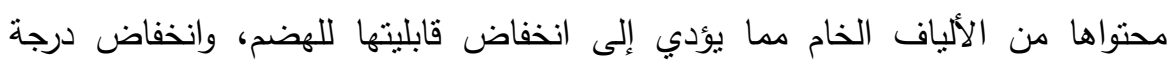
استساغتها فلا تستهلك منها الحيوانات كميات كبيرة، ولذلك تسمى هذه المخلفات بمواد العلف الخشنة الفقيرة. ولقد ترتب على تلك الخصائص أنه على الرغم من نوافرها بكميات

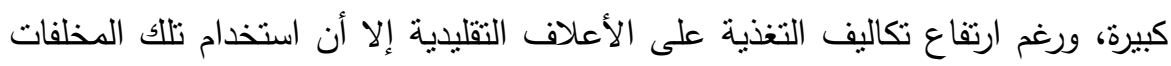
في تغذية الحيوانات يتسم بالمحدودية الأمر الذي دفع الباحثين في مجال تغذية الحيوان 
إلى إيجاد العديد من الطرق للنغلب على الخصائص السابقة لتلك المخلفات وتحسين قيمتها الغذائية، وتعظيم الإستفادة منها بواسطةالحيوان وذلك من خلال إنتاج ما يسمى على لإنى بالأعلاف الغير تقليدية. جدول رقم(ع): نطور الطاقة الإنتاجية من أهم المخلفات الزراعيـة في جمهوريـة مصر

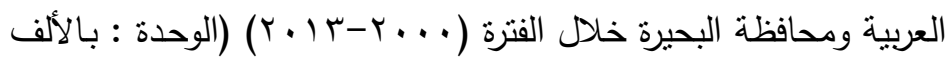

طن

\begin{tabular}{|c|c|c|c|c|c|c|c|c|c|c|}
\hline \multirow{2}{*}{$\%$} & \multicolumn{2}{|c|}{ الإجمالى } & \multirow{2}{*}{$\%$} & \multicolumn{2}{|c|}{ هُس الأزلز } & \multirow{2}{*}{$\%$} & \multicolumn{2}{|c|}{ حطب الأرةٌ الشُّامبة } & \multirow{2}{*}{ السئولك } & \multirow[b]{2}{*}{ a } \\
\hline & البمريةر & الجهورية' & & البمرية & الجمهورية' & & 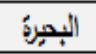 & الجمهورية' & & \\
\hline 11 & ANF & 1.19 & 17 & iri & $r \leqslant 1$. & 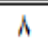 & $r \leqslant v$ & 49,7 & r.... & 1 \\
\hline $9,4 r$ & $\lambda \leqslant 1$ & 9.70 & 11 & 业Y & RANT & $\Lambda$ & 4.9 & $01 \leqslant 4$ & $r+\ldots 1$ & $T$ \\
\hline $1 \cdot, \mathrm{v}$ & MNo & ATqR & 10 & 0,0 & ודי & $\Lambda$ & rA. & \&qrV & $r+, r$ & $r$ \\
\hline 1,0 & AVI & ATM & $1 \varepsilon$ & $\{09$ & Fiv & $\Lambda$ & का & $0, \leqslant 0$ & $r \ldots r$ & 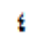 \\
\hline 1,0 & 194 & $10 \leqslant \Lambda$ & 1\{ & $\{\%$ & FrAl & $\Lambda$ & 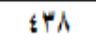 & $017 \mathrm{~V}$ & $r_{1}+\varepsilon$ & 0 \\
\hline $1, \mathrm{v}$ & 974 & 9,47 & ir & \&ा & $r+9$ & 9 & orr & ONIY & $r+10$ & 1 \\
\hline $9, \xi$ & 1.9 & 1079 & $1 \leqslant$ & $\{A 1$ & ros & $\mathrm{v}$ & THA & 0.10 & $r+.+7$ & $\mathrm{v}$ \\
\hline $1+, 1$ & 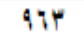 & 9.00 & 10 & 001 & rvis & $\Lambda$ & $\{+0$ & $0 \% 91$ & $r \ldots v$ & $\Lambda$ \\
\hline $11, r$ & $1.7 \%$ & 90,4 & ir & 014 & rA9؛ & 1. & $0 \leqslant 9$ & 07.9 & $r \ldots i$ & 9 \\
\hline $1 r, r$ & $1 . \times 9$ & MNOV & 10 & $\$ 4$ & 191. & 11 & ior & $09 \leqslant V$ & $r+.9$ & 1. \\
\hline $11, r$ & $1 . \leqslant 0$ & qrol & $1 \varepsilon$ & \&V7 & $r \xi, r$ & 1. & 079 & $0 \wedge \leqslant \Lambda$ & $r+1$. & 11 \\
\hline $11,\{$ & 1.11 & 940. & $1 \varepsilon$ & $\leqslant v_{0}$ & Frq & 1. & 091 & 0907 & $r .11$ & ir \\
\hline 11,0 & $1, A V$ & $q \leqslant \leqslant$ & 1\{ & 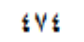 & KYAE & 1. & 314 & r.t. & $r+1 r$ & 14 \\
\hline 11,1 & 11.9 & $90 \leqslant 0$ & 1\{ & \&VT & $r Y v \varepsilon$ & 1. & וזי & $11 \times 1$ & $r+1 r$ & 14 \\
\hline 1,9 & 94,1 & $191,, r$ & 1\{ & $\leqslant \vee \Lambda, 0$ & $r \leqslant \mu$ & 9 & $\{9,1 \leqslant$ & O\&VY,Y & وبط & \\
\hline
\end{tabular}

المصدر : جمعت وحسبت من وزارة الزراعة واستصلاح الأراضي، قطاع الثشئون الإقتصادية، الإدرة المرظزية للإقتصاد الزراعي، نشرة الإحصاءات الزراعية، أعداد متفرقة . 
خامساً :المعوقات والمشاكل التي تواجه المزارعين عند محاولة الإستفادة من المخلفات

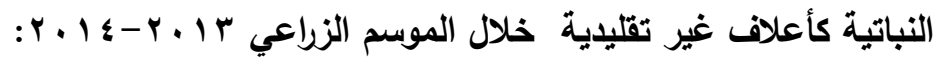

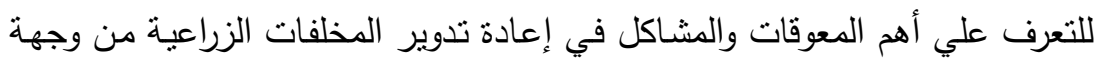

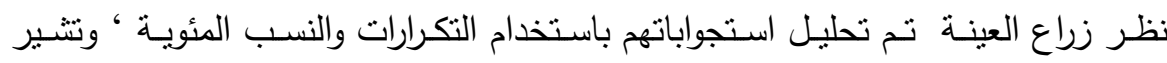
النتائج بالجدول رقم (0) إلي أن أهم المعوفات تتمثل في نقص آلات كبس قش الأرز

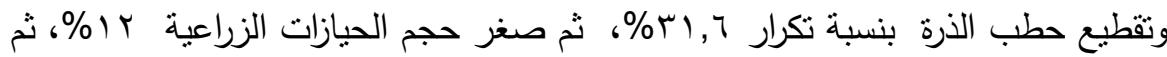

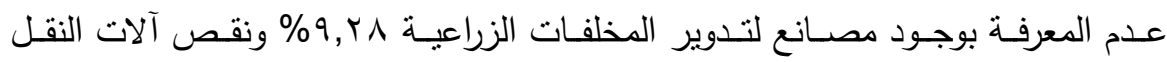
وماكينات الفرم ؟^,^\% وارتفاع تكلفة نقل المخلفات الزراعية لمكان آخر بعيدا عن الحقل

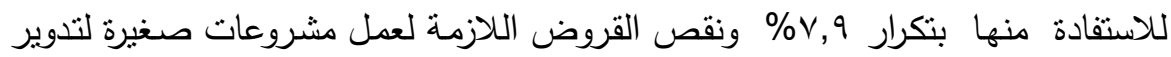

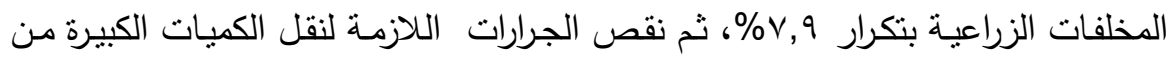

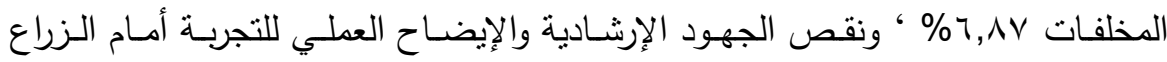
. \% $\leqslant, \wedge 1$ جدول رقـم(•): توزيع مزارعي العينـة وفقاً لأرآئهم حـول أهم المعوقات والمشـاكل التي تواجهرم عند محاولة الإستفادة من المخلفات النباتية:

\begin{tabular}{|c|c|c|}
\hline \% \% للتكرار & 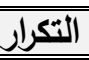 & المعوقّات \\
\hline r 1,7 & 94 & 1- نقص آلات كبس قش الأرز وتقطيع حطب الذرة \\
\hline Ir & ro & r- صغر حجم الحيازات الزراعية \\
\hline $9, \mathrm{\wedge}$ & TV & r- عدم وجود مصانع لتدوير المخلفات الزراعية \\
\hline$\Lambda, 94$ & ry & ع - نقص آلات النقل وإمكانيات الفرم \\
\hline$\vee, q$ & r & 0- ارتفاع تكلفة نقل المخلفات الزراعية \\
\hline$\vee, q$ & $r r$ & آ - نقلص القروض اللازمة لعمل مشروعات تدوير \\
\hline $7, \wedge \vee$ & r. & V- نقص الجرارات اللازمة لنقل المخلفات الزراعية \\
\hline$\varepsilon, \wedge)$ & $1 \varepsilon$ & أمام الزراع الجهود الإرشادية والإيضاح العملي بالتجربة \\
\hline
\end{tabular}

المصدر: جمعت البيانات وحسبت من استمارة الاسنبيان الزراعي بمحافظة البحيرة

$$
r \cdot 1 \varepsilon / r \cdot 1 r
$$


سادساً: المقترحات للتظلب على المعوقات والمشاكل التي تواجه المزارعين عند محاولة الإستفادة من المخلفات الزراعية كأعلاف غير تقليدية خلال الموسم الزراعي $: r+1 \leq / r \cdot 1 r$

يتضح من النتائج الواردة بالجدول رقم (T) أن نشر مصانع الأعلاف غير التقليدية في الريف المصري كمقترح لحل هذه المعوفات ياتي في المرتبة الأولي من حيث الأهمية

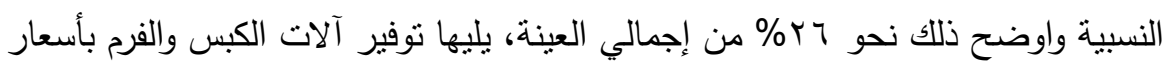

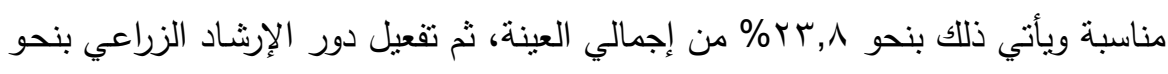

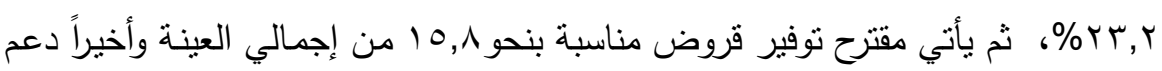

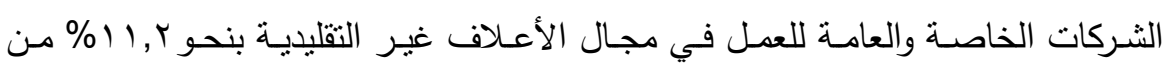
إجمالي العينة . جدول رقم(7): توزيع مزارعي العينة وفقاً لأرائهم حول مقترحاتهم للتغلب على المشاكل

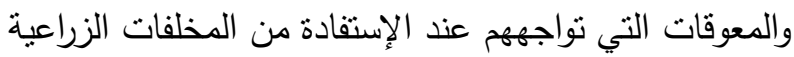

\begin{tabular}{|c|c|c|c|}
\hline تكرار \% ت ت & 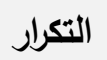 & المقترحات اللتظلب على هذه المعوقات والمشاكل & م \\
\hline ru & ir. & توفير مصانع الأعلاف غير التقليدية في الريف المصري & 1 \\
\hline$r, \Lambda$ & 11. & توفير ألالآت الكبس والفرم والتقطيع بأسعار مناسب & $r$ \\
\hline r & $1 \cdot v$ & تفعيل دور الإرشاد الزراعي & r \\
\hline 10,1 & $V T$ & توفير قروض بأسعار مناسبة & $\varepsilon$ \\
\hline $11, r$ & or & دعم الشركات العامة والخاصة للعمل في مجال الأعلاف غير & 0 \\
\hline
\end{tabular}


مجلة العلوم البيئية

معهد الدراسات والبحوث البيئية - جامعة عين شمس البه

\section{zall}

أحمد كمـال أبو ريـه(979 (19): تغذيـة الحيوان والدواجن، الأسس العلميـة الحديثة والعلائق والأعلاف، دار المعارف الطبع الثانية، القاهرة.

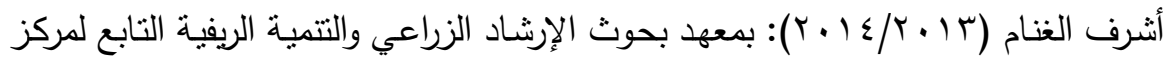

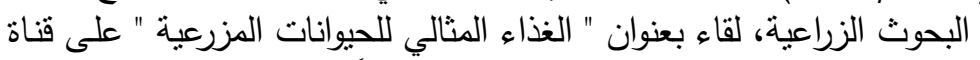

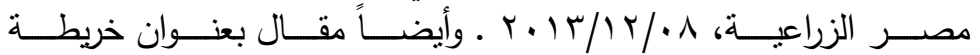

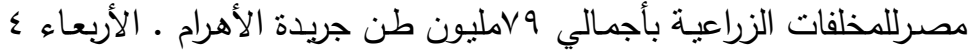

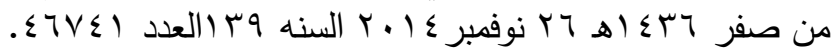

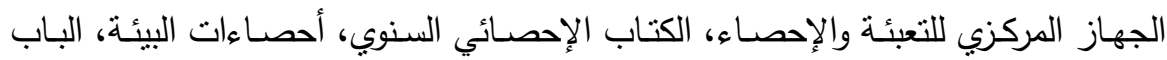

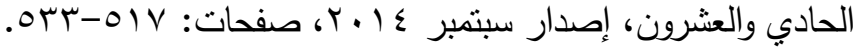

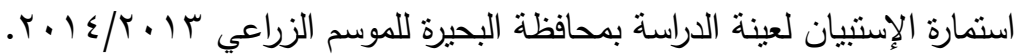

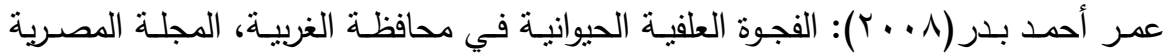

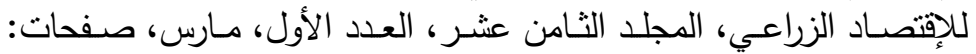

$$
.107-1 \leqslant 0
$$

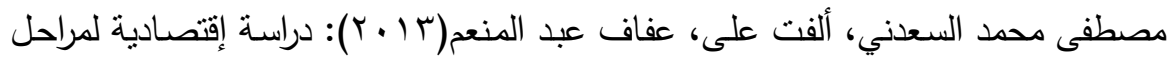

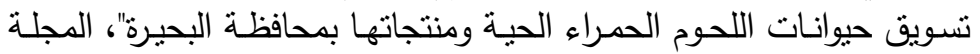

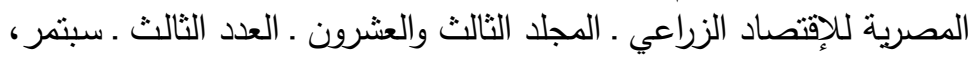

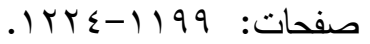

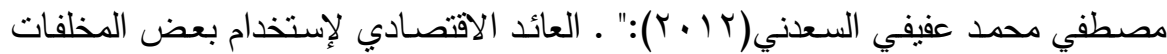

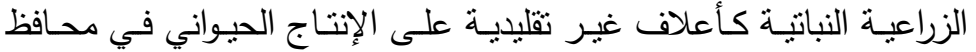

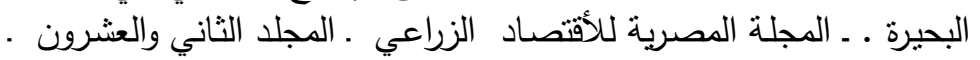

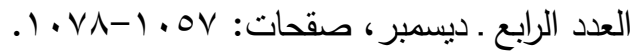

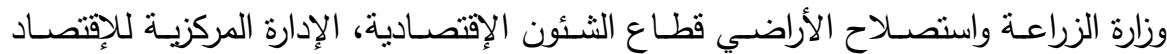
الزراعي، نشرة إحصاءات الثزوة الحيوانية، ونشرة الإحصائات الزراعية أعداد الإداد الإداد متفرقة. - مزي،

وزارة الزراعـة واستصــلاح الإراضـي، مديريـة الزارعـة بمحافظـة البحيرة ، الإرشـاد الزراعي،

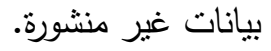


El-Giziry, A.; M. A. A. Abd El-Hady and M. A. I. Khalil (2011): Utilization of Rice Straw for feeding Ruminants : 4Economic and Nutritional Study of Corn Stalks and Green Rice Straw Silages in Partial Replacement of Berseem in Winter Feeding of Lactating Buffaloes " Egyptian J. Nutrition and Feeds. 14 (3): 433-444.

\title{
ECONOMIC STUDY OF THE USE OF SOME AGRICULTURAL WASTES AS NON- TRADITIONAL FEEDS
}

\author{
El-Tallawy, F. M. ${ }^{(1)}$; Solomon, Nayrah, Y. ${ }^{(2)}$; \\ Abd El- Hamid, Siham, A. ${ }^{(1)}$ and Kholif, A. A. ${ }^{(1)}$ \\ 1) Institute of Environmental Studies and Research, Ain Shams \\ University 2) National Research Centre.
}

\begin{abstract}
The study aimed the development of livestock numbers on the level of Egypt and the province of Beheira. It was found the relative importance of the number of cattles the level of Beheira, to maintain about $12.14 \%$ of the total number of cows on the level of Egypt (about 4.5 million head). The numbers of buffalo reached to about $10.1 \%$ of the total numbers of Buffalo (3.8 million head in Egypt) during the study period in average. The study also showed that the availability of green fodder exceeds the needs of which about 17 million tons during the period (2010-2013), where it is a green fodder needing about $68 \%$ at the level of Egypt, while the requirement of green fodder at the level of Beheira toward the province of $62.3 \%$ of the available during the period, while the deficit of concentrated feed was about 7.1 million tons at the level of

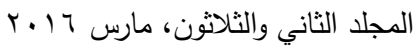


Egypt and 750 thousand tons at the level of Beheira province during the period (2010-2013).

As the volume of total agricultural wastes (rice straw, corn stover) about 8.9 million tons nationwide, including Beheira, which represented about $10.9 \%$ of the amount (968 600 tons).

Keywords: Province of Beheira, availability of green fodder, agricultural wastes, farm animals requirements 